\title{
INVESTIGACIONES
}

\section{Problemas conductuales en preescolares chilenos: Percepción de las madres y del personal educativo*}

\author{
Behavior Problems in Chilean Preschool Children: \\ Perceptions of Mothers and Educational Staff \\ Chilenos problemas de comportamento pré-escolar: \\ Percepções de mães e os professores
}

\author{
Adriana Gómez S., ${ }^{a}$ María Pía Santelices A., ${ }^{b}$ Diana Gómez G., ${ }^{c}$ \\ Claudia Rivera M., ${ }^{d}$ Chamarrita Farkas K. ${ }^{e}$ \\ ${ }^{a}$ Escuela de Psicología, Pontificia Universidad Católica de Chile. Fono: 9-88302166. \\ Correo electrónico: agomed@uc.cl

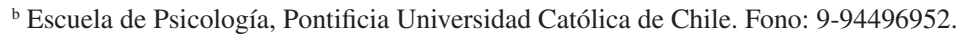 \\ Correo electrónico: msanteli@uc.cl \\ ${ }^{c}$ Escuela de Postgrado, Facultad de Ciencias Sociales, Universidad de Chile. Fono: 9-94187126. \\ Correo electrónico: dianamarcelag@gmail.com \\ ${ }^{d}$ Escuela de Psicología, Pontificia Universidad Católica de Chile. Fono: 56-2-3544639. \\ Correo electrónico: crrivera@uc.cl \\ e Escuela de Psicología, Pontificia Universidad Católica de Chile. Fono: 56-2-3544639. \\ Correo electrónico: chfarkas@uc.cl
}

\section{RESUMEN}

El desarrollo emocional y conductual de los niños preescolares se ha producido en áreas como la detección y abordaje de los problemas conductuales, lo que permite la prevención para etapas posteriores. En el presente estudio se tomó la perspectiva de diferentes informantes, investigando el acuerdo entre las madres y el personal docente respecto a las puntuaciones de los problemas conductuales en los niños. Las madres $(n=162)$ y el personal docente ( $n=42)$ evaluaron a cada niño ( $n=162,36$ a 49 meses) con el "Inventario de Problemas Conductuales y Socio-emocionales", IPCS. El acuerdo entre los informantes se analizó a través de las puntuaciones medias y pruebas t. Las puntuaciones de las madres fueron significativamente más altas que las del personal docente, informando la presencia de un nivel de riesgo o problemas de comportamiento, en contraste con los docentes que ubican a los niños en una categoría normal.
\end{abstract}

Palabras clave: acuerdo entre padres y profesores, preescolares, problemas de comportamiento.

\section{ABSTRACT}

The behavioral and emotional development in preschool children has been developed in topics like detection and approach of behavioral problems in children, allowing the prevention in later stages. In this regard, we have investigated the agreement between mothers and educational staff of Chilean preschoolers regarding scores on behavioral problems in children. Mothers $(n=162)$ and educational staff $(n=42)$ assessed each child $(n=162,36$ to 49 months) through the "Inventario de Problemas Conductuales y Socio-emocionales", IPCS. The agreement between mothers and teachers

\footnotetext{
* El artículo contó con la financiación otorgada por el Fondo Nacional de Desarrollo Científico y Tecnológico, FONDECYT,
} $\mathrm{N}^{\circ} 1100721$. 
was analyzed through mean scores and t-tests. The mean scores rated by mothers were significantly higher than those of educational staff, the scores from the maternal perspective correspond to the level of risk or behavioral problems, in contrast to the perception of the educational staff whose mean falls under the category of normal.

Key words: agreement parents and teachers, preschoolers, behavioral problems.

\section{RESUMO}

O desenvolvimento comportamental e emocional em crianças pré-escolares teve uma frente prolífica na pesquisa, especificamente no que diz respeito à detecção e à abordagem de problemas comportamentais nesta fase, o que permite a prevenção de problemas em fases posteriores. A este respeito, investiga-se o acordo entre mães e pessoal docente de pré-escolares do Chile com relação aos escores de problemas comportamentais em crianças. As mães $(n=162)$ e de pessoal docente $(n=42)$ avaliados cada criança $(n=162,36$ a 49 meses), por meio do "Inventário de Problemas Conductuales y Sócio-emocionales", IPCS. O acordo entre mães e professores foi analisado por meio de dois indicadores, a pontuação média e t-testes. Os escores médios avaliados por mães foram significativamente maiores do que os de pessoal docente; a pontuação a partir da perspectiva materna corresponde ao nível de risco ou problemas de comportamento, ao contrário da percepção do pessoal docente, cuja média se enquadra na categoria de normal.

Palavras chave: acordo de pais e professores, pré-escolares, problemas comportamentais.

\section{INTRODUCCIÓN}

El interés por el desarrollo socio-emocional de los niños ha aumentado considerablemente en las últimas décadas con el surgimiento de nuevas líneas de investigación, específicamente en los problemas conductuales y emocionales en la población preescolar (Hinshaw et al., 1992), la cual se constituye como un período clave para la identificación de manifestaciones tempranas de conductas problemáticas.

Estudios con niños escolares y adolescentes estiman que la prevalencia de los trastornos de conducta en la población general sería del 5 al 10\% (Moffitt \& Scott, 2008), siempre con un predominio significativo del sexo masculino respecto al femenino de 2,5:1 (Moffitt \& Scott, 2008). Dentro de las consultas psiquiátricas infantiles el trastorno oposicionista desafiante (TOD) y el trastorno disocial (TD) representan cerca del 12\% (Almonte, Montt y Correa, 2003).

En Chile, con respecto al TOD, se ha encontrado una prevalencia en la población general de un 7,6 \% (De la Barra, Toledo y Rodríguez, 2002). Éste suele darse con mayor frecuencia en la etapa escolar, entre 6 y 8 años (Almonte, Montt y Correa, 2003), mientras que el TD tiene una prevalencia del 5 al $9 \%$ en la población general (Rioseco et al., 2009).

En la literatura son escasos los estudios en preescolares propiamente, por lo que es una necesidad saber a nivel general cómo se dan los problemas conductuales en la infancia temprana en Chile, cuáles son los más comunes y cómo deben evaluarse y medirse. Esto llevaría a desarrollar programas dirigidos a atender dichos problemas tempranamente, los cuales influirían a corto plazo en mejorar la adaptación y felicidad del niño y su familia, y en prevenir psicopatología a largo plazo, ya que es conocida la correlación existente entre la psicopatología infantil y la adulta (CEDEP, 1983).

Es por esto que en el presente estudio se examinará la percepción de los problemas conductuales de niños en edad preescolar, reportada por dos adultos significativos: su madre y la educadora del jardín infantil al que asiste, ya que dos perspectivas permiten complejizar la visión sobre ellos, lo cual tiene implicaciones para la clínica donde es necesario observar la conducta del niño en diferentes contextos (casa-jardín infantil). A su vez, es importante señalar las diferencias entre ambos informantes, con el fin de conocer las tendencias de cada uno y así generar marcos comprensivos más amplios. 


\section{PROBLEMAS CONDUCTUALES EN PREESCOLARES}

Los problemas conductuales pueden describirse como un conjunto de dificultades socio-emocionales que se manifiestan en diferentes conductas de niños y niñas, y que suelen generar dificultades entre los padres y los hijos, con los pares, entre otros. Pueden ser clasificados en dos amplios dominios: los problemas internalizantes y los externalizantes. Las dificultades como la ansiedad, miedos, retraimiento y timidez se encuentran dentro de las dificultades internalizantes, mientras que las externalizantes incluyen agresión, hiperactividad, impulsividad e inatención (Briggs-Gowan et al., 2006).

Durante la infancia, los problemas de conducta provocan un malestar considerable para los niños, sus familias y sus establecimientos educativos, asociados con consiguientes deficiencias sociales y educativas para los niños (Lahey et al., 1997 Cit. en Moffitt \& Scott, 2008). Por lo tanto, la identificación adecuada de los niños en situación de riesgo para el desarrollo de trastornos emocionales y conductuales, e incluso la detección de los problemas conductuales, es uno de los componentes claves para el establecimiento de programas de prevención para la infancia.

Los problemas conductuales pueden revelarse desde períodos tempranos del desarrollo, estudios en población general hablan del 5-10\% y además muestran una fuerte estabilidad, Moffitt \& Scott (2008) retoman resultados de estudios longitudinales donde indican que entre el 50 y $60 \%$ de los niños que presentan altos índices de mal comportamiento a la edad de los 3 a 4 años seguirán mostrándolos en la edad escolar. Por lo tanto, se plantea que estas dificultades tienen permanencia y estabilidad en el tiempo, perpetuando las dificultades en la edad escolar e incluso en la adultez (Briggs-Gowan et al., 2006; Campbell, Shaw \& Gilliom, 2000; Feeney-Kettler, Kratochwill \& Kettler, 2011). Además, han sugerido que la estabilidad puede ser más coherente y más fuerte para los problemas de externalización que para los internalizantes (Briggs-Gowan et al., 2006; Mesman, Bongers \& Koot, 2001).

Según el estudio realizado en población preescolar chilena en Santiago por Seguel et al. (1992), se estima que un 29,7\% de niños presenta alteraciones conductuales, mostrando agresividad, retraimiento, inmadurez e imagen disminuida.

\section{EVALUACIÓN DE PROBLEMAS CONDUCTUALES}

Los niños deben ser considerados como continuamente cambiantes en relación con su desarrollo, por lo tanto, detectar un problema de conducta es una tarea difícil, dado que una conducta puede ser normal o no dependiendo de la etapa de desarrollo de cada niño y de qué tanto se adapte a las condiciones ambientales. De hecho, algunas conductas problemáticas pueden movilizar procesos de desarrollo que culminan en niveles superiores de adaptación.

Así mismo, la evaluación de niños debe considerar su inclusión en un ambiente familiar, educativo o social, ya que desde temprana edad los niños interactúan con otros, produciéndose una relación transaccional entre sus características individuales y el contexto en el que funcionan. En este sentido, las evaluaciones generalmente requieren la perspectiva de un adulto para determinar qué tanto puede afectar una conducta a un niño en su proceso de desarrollo.

Sin embargo, un tipo de conducta puede ser más conflictiva para un tipo de adulto que para otro, dependiendo de las normas, valores, la historia personal y el medio sociocultural al que pertenece; esa perspectiva de los adultos influirá en lo que se considere problemático o no 
respecto de un niño (CEDEP, 1983). Es así como una misma conducta de un niño en diferentes contextos puede ser leída de forma distinta por los adultos que lo rodean, dando lugar a las discrepancias y concordancias en la información suministrada por diferentes observadores.

Se han realizado varios estudios sobre este tema (Achenbach, 2011; De los Reyes et al., 2011; Dirks, Boyle \& Georgiades, 2011; Medina et al., 2007; Satake et al., 2003), encontrándose diferencias significativas entre cada grupo de evaluadores. Uno de estos estudios con adolescentes evaluados con la escala de problemas conductuales de Achenbach (2011), tanto desde la perspectiva del personal educativo, padres y el auto-reporte, concluye que existe una concordancia baja en las escalas de problemas internalizantes y una concordancia un poco más alta en las subescalas de atención, comportamiento agresivo, externalizantes y puntaje total. También se observó que al informar sobre quejas somáticas, problemas de pensamiento y atención reportan más altos puntajes los padres, seguidos por los adolescentes y por último los maestros; igualmente, los padres indican mayores problemas de retraimiento en adolescentes, seguidos por el personal educativo y por último los propios adolescentes. Finalmente, en las escalas de depresión/ansiedad, problemas sociales, conductas delictivas, agresividad y externalizantes, los adolescentes informaron más alteraciones, seguidos por los padres y por último el personal educativo (Medina et al., 2007).

Otro estudio con una muestra de niños japoneses evaluados con el Child Behavior Checklist, indica que los padres y los profesores los evalúan de forma diferente, siendo los padres quienes detectan más problemas conductuales en comparación con el personal educativo (Satake et al., 2003) lo cual coincide con otros estudios que concluyen en el mismo aspecto (Winsler \& Wallace, 2002).

De los Reyes \& Kazdin (2004) sustentan que posiblemente estas discrepancias entre informantes pueden provenir de las diferentes características de cada observador. No obstante, no es posible determinar cuál de estas informaciones es la mejor, o cuál de los informantes es el más preciso.

Por otra parte, las percepciones de los problemas conductuales según el género de los niños también han sido investigadas. Estudios plantean que tanto padres como profesores perciben una mayor cantidad de problemas externalizantes en niños que en niñas (Touliatos \& Lindholm, 1981; Winsler \& Wallace, 2002).

Las investigaciones se han interesado constantemente por tratar de determinar el grado de correspondencia entre los diferentes adultos informantes con respecto a la evaluación de los problemas conductuales (Hinshaw et al., 1992), otros proponen que dichas concordancias pueden surgir como errores en la medición de dichas conductas (De los Reyes, 2011). Sin embargo, más allá de pensar las divergencias como un error de medida o como un obstáculo para la evaluación, se ha comenzado a discutir sobre lo que implican dichas diferencias.

Achenbach (2011) sostiene que diferentes informaciones o reportes sobre un mismo niño no deberían ser consideradas informaciones erróneas, sino que deberían entenderse como las diferentes formas de funcionamiento del ser humano en diferentes campos. Adicionalmente, proporcionan información relevante sobre las conductas de los niños, por ejemplo, el tiempo de aparición, las características según el contexto, la expresión comportamental particular, y permiten determinar cómo será la evolución de la conducta en cuestión por la posibilidad de acceder a diferentes perspectivas (a diferencia de los reportes de un solo informante) y, por lo tanto, identificar objetivos de atención en la clínica (De los Reyes, 2011). Otra razón de la importancia de la existencia de dichas discrepancias es que reflejan cómo la genética y el ambiente influyen en la conducta del niño (Achenbach, 2011). 
En general, las discrepancias - más que ser consideradas errores en la medición- ayudan a incrementar el marco de comprensión de las causas y consecuencias de los problemas conductuales y sus posibles tratamientos (De los Reyes, 2011). Y es hacia este punto que avanzan las investigaciones actuales, a diferencia de las investigaciones más antiguas que concluían sobre las debilidades de padres o profesores para ponerse de acuerdo como informantes sobre la conducta de los niños (Achenbach, 2011).

\section{MÉTODO}

\subsection{DISEÑO}

El diseño está adscrito a los lineamientos de la investigación cuantitativa, correspondiendo a un diseño no-experimental, de tipo descriptivo-correlacional y de carácter transversal.

\subsection{PARTICIPANTES}

En el estudio participaron 162 díadas madre-hijo, además del personal educativo a cargo $(n=42)$ de estos niños en sus correspondientes jardines infantiles. Las díadas se conformaron por un grupo de 162 madres cuya edad promedio fue de 30,04 años (D.S=7,06) en un rango desde 18 a 49 años, quienes fueron evaluadas con sus hijos, con una edad promedio de 41,72 meses (D.S.=2,33), 59,9\% (97) de ellos son varones, y el 40,1\% (65) mujeres. La selección de los 27 jardines infantiles se realizó bajo un muestreo aleatorio, a partir de los jardines infantiles del sistema público de la Región Metropolitana en Santiago de Chile. Para la selección de los niños se utilizó como criterio de inclusión la edad en un rango de entre 39 y 44 meses y la asistencia regular al jardín infantil, y como criterio de exclusión se definió la presencia de alguna enfermedad grave o discapacidad severa. Ver la Tabla 1 y la Tabla 2 para conocer las características de la muestra, la cual pertenece al proyecto Fondecyt No 1100721.

Tabla 1. Antecedentes de los niños de la muestra

\begin{tabular}{|l|l|l|l|l|}
\hline Nombre & Promedio & $\begin{array}{l}\text { Desviación } \\
\text { Estándar }\end{array}$ & Mínimo & $\begin{array}{l}\text { Rango } \\
\text { Máximo }\end{array}$ \\
\hline Edad gestacional & 38,42 semanas & 2,54 & 30 semanas & 43 semanas \\
\hline Peso al nacer & 3411,43 gramos & 617,95 & 1240 gramos & 5000 gramos \\
\hline Talla al nacer & $49 \mathrm{~cm}$. & 3,96 & $28,5 \mathrm{~cm}$. & $55 \mathrm{~cm}$. \\
\hline Tiempo de lactancia & 16,06 meses & 11,38 & 1 mes & 45 meses \\
\hline Edad de ingreso a la sala cuna & 18,59 meses & 11,36 & 2 meses & 39 meses \\
\hline $\begin{array}{l}\text { Horas semanales que el niño } \\
\text { pasa en el jardín }\end{array}$ & 31,32 horas & 14,38 & 4 horas & 55 horas \\
\hline
\end{tabular}

Fuente: elaboración propia. 
Tabla 2. Antecedentes de las madres de la muestra

\begin{tabular}{|c|c|c|}
\hline \multicolumn{2}{|l|}{ Antecedente } & $\begin{array}{l}\text { Madre } \\
\begin{array}{l}\text { Porcentaje de madres / } \\
\text { (frecuencia) }\end{array}\end{array}$ \\
\hline \multirow{3}{*}{ Nacionalidad } & Chilena & $075 \%(119)$ \\
\hline & Peruana & $1,6 \%(2)$ \\
\hline & Argentina & $0,8 \%$ (1) \\
\hline \multirow{6}{*}{$\begin{array}{l}\text { Nivel } \\
\text { educacional }\end{array}$} & Básica incompleta o inferior & $5,1 \%(8)$ \\
\hline & Básica completa & $10,8 \%(38)$ \\
\hline & Media incompleta & $24,1 \%(38)$ \\
\hline & Media completa-Educación técnica incompleta & $35,4 \%(56)$ \\
\hline & $\begin{array}{l}\text { Universitaria incompleta o Instituto Profesional } \\
\text { incompleto }\end{array}$ & $15,8 \%(25)$ \\
\hline & Universitaria completa & $8,9 \%(14)$ \\
\hline \multirow{3}{*}{$\begin{array}{l}\text { Situación } \\
\text { ocupacional }\end{array}$} & Cesante o no trabaja & $35,6 \%(53)$ \\
\hline & Estudia & $5,4 \%(8)$ \\
\hline & Trabaja & $59,1 \%(88)$ \\
\hline
\end{tabular}

Fuente: elaboración propia.

\subsection{INSTRUMENTOS}

El Inventario de Problemas Conductuales y Socio-emocionales, I.P.C.S. (Rodríguez et al., 2000) se utilizó para evaluar los problemas conductuales y socio-emocionales en los niños y las niñas del estudio. Es una prueba de tamizaje diseñada para identificar problemas conductuales y/o socio-emocionales en niños y niñas de 3 a 5 años. Este instrumento ha demostrado tener sólidas características psicométricas, un alto nivel de validez y confiabilidad. Está diseñado para ser completado por la madre (o un adulto significativo para el menor), en forma individual, frente a un entrevistador previamente capacitado. Consta de tres partes o secciones, una dirigida a detectar los problemas en el niño, otra parte para detectar los problemas de la madre, y una última parte referida a situaciones familiares estresantes.

En este estudio se consideró únicamente la primera parte (Sección Niño), en la que el adulto debe responder frente a cada pregunta si la conducta se presenta siempre, a veces o nunca. Tiene una puntuación de 0 a 2 puntos por ítem, siendo el puntaje máximo de 90 puntos. El tiempo de administración es de 20 minutos aproximadamente. El puntaje total de cada sección y por factores se transforma a percentiles, permitiendo obtener un perfil de cada sección. De acuerdo a los objetivos de este estudio, este instrumento fue contestado por la madre y por el personal educativo encargado del niño en el jardín infantil. La confiabilidad del instrumento en esta muestra en la Sección Niño fue adecuada, tanto para los apoderados como para el personal educativo $(\alpha=, 735$ en la aplicación a los apoderados y $\alpha=, 790$ en la aplicación al personal educativo). 
Los factores que componen este instrumento son Agresividad, Retraimiento, Inmadurez, Comportamiento Extraño, Control de Esfínteres, Ansiedad, Imagen Disminuida, Comportamiento según Género y Temores. Factores que describen síntomas internalizantes y externalizantes.

\subsection{PROCEDIMIENTO}

Posterior al contacto telefónico con los jardines infantiles y la entrega y aprobación de los consentimientos informados a los directores y el personal del jardín, éstos proporcionaron una lista con los niños que cumplían con los requisitos solicitados para el estudio y a partir de allí se contactaron las madres. Las evaluaciones fueron realizadas por una dupla de evaluadores que tuvo una capacitación de 12 horas, en las que se revisaron los instrumentos que se aplicarían a los niños y los adultos. La administración de los instrumentos se realizó por separado a los adultos (madre y personal educativo).

\section{ANÁLISIS DE DATOS}

Se realizaron análisis descriptivos para obtener una perspectiva global de la muestra evaluada según la variable problemas conductuales. Luego, se aplicaron pruebas t para muestras independientes, para identificar diferencias entre las percepciones de la madre y la del personal educativo y Coeficiente de Chi cuadrado, para observar relaciones entre ambas percepciones en las variables categoriales.

\section{RESULTADOS}

\subsection{ANÁLISIS DESCRIPTIVOS}

Según la perspectiva de las madres de la muestra respecto a la presencia de problemas conductuales en los niños, se da un promedio de 12,96 puntos (D.S.=7,31), que corresponde a la categoría de riesgo o con problemas. Considerando la muestra general, el 56,5\% (91) de los niños no presenta problemas conductuales, mientras que el 43,5\% (71) presenta problemas conductuales. En cuanto a los factores de análisis de los problemas conductuales, tal como se observa en la Tabla 3, la mayoría se encuentra en la categoría de normalidad, a excepción de los factores Inmadurez y Comportamiento Extraño que, según las madres, son las áreas en las que los niños presentarían mayores dificultades de problemas conductuales.

Tabla 3. Puntajes y categorías de los factores de problemas conductuales y socio-emocionales según madres

\begin{tabular}{|l|l|c|c|c|}
\hline $\mathbf{N}^{\mathbf{0}}$ & Factor & Promedio & D.S. & Categoría \\
\hline I & Agresividad & 2,43 & 2,52 & Normal \\
\hline II & Retraimiento & 1,32 & 1,84 & Normal \\
\hline III & Inmadurez & 1,79 & 1,72 & Riesgo o con problemas \\
\hline
\end{tabular}




\begin{tabular}{|l|l|c|c|c|}
\hline IV & Comportamiento Extraño & 0,85 & 1,35 & Riesgo o con problemas \\
\hline V & Control de Esfínteres & 0,84 & 1,09 & Normal \\
\hline VI & Ansiedad & 2,25 & 1,92 & Normal \\
\hline VII & Imagen Disminuida & 0,39 & 0,76 & Normal \\
\hline VIII & Comportamiento según Género & 0,44 & 0,8 & Normal \\
\hline IX & Temores & 2,54 & 1,66 & Normal \\
\hline
\end{tabular}

Fuente: elaboración propia.

Por otra parte, en la percepción del personal educativo se da un promedio de 8,77 puntos (D.S.=7,83) lo que corresponde a la categoría de normalidad. En general, la muestra total de niños, el 79,6\% (129) no presenta problemas conductuales, mientras que el 20,4\% (33) se encuentra en la categoría de riesgo o con problemas. En cuanto al análisis por factor, la Tabla 4 muestra los puntajes y categorías de los problemas conductuales según el personal educativo. Según el personal educativo, el factor de Inmadurez es el único de la lista de factores que se encuentra en una categoría de riego o con problemas.

Tabla 4. Puntajes y categorías de los factores de problemas conductuales y socio-emocionales según personal educativo

\begin{tabular}{|l|l|c|c|c|}
\hline $\mathbf{N}^{\mathbf{1}}$ & Factor & Promedio & D.S. & Categoría \\
\hline I & Agresividad & 1,92 & 3,04 & Normal \\
\hline II & Retraimiento & 1,35 & 1,64 & Normal \\
\hline III & Inmadurez & 1,8 & 1,77 & Riesgo o con problemas \\
\hline IV & Comportamiento Extraño & 0,54 & 1,01 & Normal \\
\hline V & Control de Esfínteres & 0,36 & 0,95 & Normal \\
\hline VI & Ansiedad & 1,39 & 1,79 & Normal \\
\hline VII & Imagen Disminuida & 0,23 & 0,56 & Normal \\
\hline VIII & Comportamiento según Género & 0,19 & 0,56 & Normal \\
\hline IX & Temores & 1,28 & 1,31 & Normal \\
\hline
\end{tabular}

Fuente: elaboración propia.

\subsection{ANÁLISIS COMPARATIVOS ENTRE LA PERCEPCIÓN DE MADRES Y PERSONAL EDUCATIVO}

A partir de los análisis realizados, se observan diferencias significativas entre el puntaje total de problemas conductuales, otorgado por las madres y otorgado por el personal educativo $(t=4,98 ; p \leq .01)$, siendo mayor el puntaje otorgado por las madres respecto de los problemas conductuales observados en los niños (ver Tablas 5 y 6). En cuanto a la categoría del puntaje de cada una de las percepciones, se observan relaciones estadísticamente significativas $\left(\chi^{2}=18,8 ; p \leq .01\right)$, ya que el personal educativo percibe 
a una mayor cantidad de niños en la categoría de normalidad, en comparación con las madres. Por su parte, las madres perciben a una mayor cantidad de niños en categoría de riesgo respecto de los problemas conductuales, a diferencia del personal educativo.

Tabla 5. Estadísticos de grupo

\begin{tabular}{|c|c|c|c|c|c|}
\hline & Tipo de adulto & $\mathbf{N}$ & Media & $\begin{array}{l}\text { Desviación } \\
\text { típ. }\end{array}$ & $\begin{array}{l}\text { Error típ. } \\
\text { de la media }\end{array}$ \\
\hline \multirow{2}{*}{$\begin{array}{l}\text { Puntaje Factor I IPCS } \\
\text { sección niño mamá }\end{array}$} & Apoderado & 162 & 2,43 & 2,524 & ,198 \\
\hline & Personal Educativo & 162 & 1,92 & 3,040 & 239 \\
\hline \multirow{2}{*}{$\begin{array}{l}\text { Puntaje Factor II IPCS } \\
\text { sección niño mamá }\end{array}$} & Apoderado & 162 & 1,32 & 1,840 &, 145 \\
\hline & Personal Educativo & 162 & 1,35 & 1,647 &, 129 \\
\hline \multirow{2}{*}{$\begin{array}{l}\text { Puntaje Factor III IPCS } \\
\text { sección niño mamá }\end{array}$} & Apoderado & 162 & 1,79 & 1,725 &, 135 \\
\hline & Personal Educativo & 162 & 1,80 & 1,779 &, 140 \\
\hline \multirow{2}{*}{$\begin{array}{l}\text { Puntaje Factor IV IPCS } \\
\text { sección niño mamá }\end{array}$} & Apoderado & 162 & 85 & 1,352 &, 106 \\
\hline & Personal Educativo & 162 & ,54 & 1,010 & ,079 \\
\hline \multirow{2}{*}{$\begin{array}{l}\text { Puntaje Factor V IPCS } \\
\text { sección niño mamá }\end{array}$} & Apoderado & 162 & 84 & 1,097 & 086 \\
\hline & Personal Educativo & 162 & ,36 & ,956 & 075 \\
\hline \multirow{2}{*}{$\begin{array}{l}\text { Puntaje Factor VI IPCS } \\
\text { sección niño mamá }\end{array}$} & Apoderado & 162 & 2,25 & 1,924 & ,151 \\
\hline & Personal Educativo & 162 & 1,39 & 1,798 &, 141 \\
\hline \multirow{2}{*}{$\begin{array}{l}\text { Puntaje Factor VII IPCS } \\
\text { sección niño mamá }\end{array}$} & Apoderado & 162 & ,39 &, 766 &, 060 \\
\hline & Personal Educativo & 162 & ,23 & ,560 &, 044 \\
\hline \multirow{2}{*}{$\begin{array}{l}\text { Puntaje Factor VIII IPCS } \\
\text { sección niño mamá }\end{array}$} & Apoderado & 162 & ,44 & 804 & ,063 \\
\hline & Personal Educativo & 162 & , 19 &, 563 &, 044 \\
\hline \multirow{2}{*}{$\begin{array}{l}\text { Puntaje Factor IX IPCS } \\
\text { sección niño mamá }\end{array}$} & Apoderado & 162 & 2,54 & 1,669 &, 131 \\
\hline & Personal Educativo & 162 & 1,28 & 1,310 & 103 \\
\hline \multirow{2}{*}{$\begin{array}{l}\text { Puntaje Total Niño IPCS } \\
\text { mamá }\end{array}$} & Apoderado & 162 & 12,96 & 7,314 & ,575 \\
\hline & Personal Educativo & 162 & 8,77 & 7,836 & ,616 \\
\hline
\end{tabular}

Fuente: elaboración propia.

Tabla 6. Resultados Pruebas T para muestras independientes

\begin{tabular}{|l|c|c|}
\hline Ítem & Puntaje T & Sig. Bilateral \\
\hline Puntaje Factor I IPCS sección niño & 1,650 &, 100 \\
\hline Puntaje Factor II IPCS sección niño &,- 159 &, 874 \\
\hline Puntaje Factor III IPCS sección niño &,- 063 &, 949 \\
\hline Puntaje Factor IV IPCS sección niño & 2,374 &, 018 \\
\hline Puntaje Factor V IPCS sección niño & 4,210 &, 000 \\
\hline Puntaje Factor VI IPCS sección niño & 4,147 &, 000 \\
\hline Puntaje Factor VII IPCS sección niño & 2,152 &, 032 \\
\hline Puntaje Factor VIII IPCS sección niño & 3,282 &, 001 \\
\hline Puntaje Factor IX IPCS sección niño & 7,554 &, 000 \\
\hline Puntaje Total Niño IPCS & 4,984 &, 000 \\
\hline
\end{tabular}

Fuente: elaboración propia. 
En cuanto al análisis por factor de problema conductual y socio-emocional, se observan diferencias en las percepciones de ambos agentes en 6 de los 9 factores de análisis, específicamente en los factores de Comportamiento Extraño $(t=2,37 ; p \leq .05)$, Control de Esfínteres $(t=4,21 ; p \leq .01)$, Ansiedad $(t=4,14 ; p \leq .01)$, Imagen Disminuida $(t=2,15 ; p \leq .05)$, Comportamiento Extraño $(t=3,28 ; p \leq .01)$ y Temores $(t=7,55 ; p \leq .01)$. En cada uno de ellos, la percepción de problemas conductuales por parte de las madres es mayor que la percepción que tiene el personal educativo (Ver Tablas 5 y 6).

En cuanto a las percepciones de normalidad o de riesgo según las madres y el personal educativo, se presentan relaciones estadísticamente significativas entre ambas, en el factor Control de Esfínteres $\left(\chi^{2}=12,78 ; p \leq .01\right)$, observándose que el personal educativo percibe una mayor cantidad de niños en la categoría de normalidad, y una muy baja cantidad de niños en riesgo, a diferencia de lo que ocurre con las madres, que perciben una mayor cantidad de niños en riesgo o con problemas en este factor. También se presentan relaciones estadísticamente significativas entre las percepciones en el factor Ansiedad $\left(\chi^{2}=7,023 ; p \leq .01\right)$, Comportamiento Extraño $\left(\chi^{2}=5,88 ; p \leq .05\right)$ y Temores $\left(\chi^{2}=33,69 ; p \leq .01\right)$, en las cuales se observa la relación anteriormente señalada: el personal educativo percibe a una mayor cantidad de niños en la categoría de normalidad, y una baja cantidad en riesgo, mientras que las madres, frente al mismo factor, perciben una mayor cantidad de niños en riesgo o con problemas.

Por otra parte, tanto el personal educativo como las madres presentan percepciones similares en los factores de Agresividad, Retraimiento e Inmadurez respecto de los niños, en los cuales no se observan diferencias estadísticamente significativas entre ambos puntajes (ver Tablas 5 y 6 ).

Respecto del género de los niños, se observa que las madres perciben mayores problemas conductuales en los niños que en las niñas $(t=-2.736 ; p \leq .005)$, específicamente en las áreas de Agresividad ( $t=-2,930 ; p \leq .005)$, Control de Esfínteres $(t=-2,508 ; p \leq .005)$ y Ansiedad $(t=-2,473 ; p \leq .005)$. En el caso del personal educativo ocurre similar situación, perciben en los niños varones mayores problemas conductuales $(t=-2,125 ; p \leq .005)$, principalmente en el área de Agresividad $(t=-3,302 ; p \leq .001)$.

En cuanto a la percepción de problemas conductuales en los niños varones, se observan diferencias estadísticamente significativas en la percepción de las madres y del personal educativo $(t=5,682 ; p \leq .001)$. Las madres perciben mayores problemas conductuales que el personal educativo en los niños, en el área de Agresividad $(t=2,553 ; p \leq, 005)$, Comportamiento Extraño $(t=2,492 ; p \leq .005)$, Control de Esfínteres $(t=5,149 ; p \leq .001)$, Ansiedad $(t=4,204 ; p \leq .001)$, Imagen Disminuida $(t=2,415 ; p \leq .005)$, Comportamiento de Género $(t=3,376 ; p \leq .001)$ y Temores $(t=5,382 ; p \leq .001)$.

En cuanto a la percepción de problemas conductuales en las niñas, se observan diferencias significativas entre las madres y el personal educativo, específicamente en el área de Temores $(t=5,32 ; p \leq .001)$. En este aspecto, las madres perciben mayores problemas conductuales en comparación con el personal educativo.

\section{CONCLUSIONES Y DISCUSIÓN}

El presente estudio examinó la consistencia o diferencia entre las madres y el personal educativo evaluando los problemas conductuales y socio-emocionales del mismo niño, utilizando el instrumento IPCS. Las madres de los sujetos evaluados encontraron mayores 
niveles de riesgo y/o problemas conductuales, con una prevalencia del 43,5\% de niños con problemas conductuales, mientras que las puntuaciones del personal educativo correspondieron a la categoría normal, con una prevalencia del 20,4\% de niños con problemas conductuales. Esta tendencia es consistente con los estudios previos (De los Reyes, 2011; Medina et al., 2007; Satake et al., 2003). El porcentaje de prevalencia de problemas conductuales de los niños, según las madres, es mayor que la prevalencia que existe en Chile, del 29,7\% (Seguel et al., 1992), siendo la prevalencia que determina el personal educativo la que se acerca más a la establecida. En este sentido, es posible plantear que esta diferencia aluda a los contextos en que ambos agentes se encuentran; en el caso de las educadoras, en el contexto educativo existe una mayor cantidad de niños con los cuales pueden comparar su desenvolvimiento y probablemente tienden a normalizar ciertas conductas en ellos. En cambio, en el caso de las madres, la comparación más que normalizar, puede tender a resaltar aquellos aspectos diferentes en sus hijos respecto de otros, considerando las expectativas que se tiene de ellos, sobre todo si es el primogénito, como ocurre en la mayor parte de esta muestra.

Al revisar cada uno de los factores que componen el I.P.C.S., se encontró que ambas visiones coincidían en el factor Inmadurez dentro de la categoría riesgo. En esta dirección, podría plantearse la posible influencia que pueda tener el personal educativo en la percepción de los apoderados, en cuanto a los estándares de desarrollo y aprendizaje, en tanto es el jardín el contexto en donde se evalúan estas características, dada la comparación con otros niños y lo altamente visible de este aspecto. En esta línea, tal y como plantean Winsler \& Wallace (2002) al observar las competencias y habilidades de los niños en el contexto educativo, el reporte más certero es el que otorga el profesor y, por ende, puede existir una influencia de esa percepción en la percepción de la madre.

Dentro de las evaluaciones de las madres se detectó que percibían a los niños con mayor problemática en los factores de Ansiedad, Comportamiento Extraño y Temores, los cuales corresponderían a síntomas clasificados como internalizantes. Mientras que, contrariamente, el personal educativo detecta mínimos niveles de riesgo en tales factores. Esto podría explicarse por el hecho de que las madres comparten con los niños un espacio más íntimo, que propicia la observación de conductas de tipo internalizante, mientras que la educadora ve al niño(a) en un contexto social, donde el foco de atención está orientado a la adaptación del niño a este contexto. De ahí que, probablemente, para el personal educativo aquellos problemas que puedan dificultar el proceso de adaptación y de enseñanza-aprendizaje sean más llamativos, correspondiendo a síntomas predominantemente externalizantes.

Respecto del género de los niños, los resultados indican que tanto las madres como el personal educativo perciben mayores problemas conductuales en los niños que en las niñas, específicamente en dos problemas externalizantes como la Agresividad y el Comportamiento Extraño, lo cual coincide con lo que plantean las investigaciones (Winsler $\&$ Wallace, 2002). De este modo, la externalización de las conductas en la etapa preescolar es mayormente observada en los niños que en las niñas, debido a las características físicas, emocionales y sociales de los niños en comparación con las niñas, en quienes se perciben problemas internalizantes como los Temores.

En general, las diferencias entre ambos agentes pueden explicarse también considerando la historia, los valores y la cultura de quien evalúa a los niños, puesto que tiene gran peso a la hora de determinar qué conducta es considerada problemática o no, por lo tanto, el factor subjetivo de cada informante tiene un lugar significativo en la percepción. 
Es importante considerar que estas diferencias en las evaluaciones también pueden responder a las distintas formas de funcionar de un sujeto en diversos contextos, por consiguiente, más que plantearse como errores en la medición o dudar sobre la validez de los reportes, dichas diferencias dan claves al clínico sobre cómo focalizar la atención y en qué ámbitos, teniendo en cuenta que la relación que establece un niño con alguno de los informantes puede ser un factor que influya en el reporte, constituyendo un campo abierto para la investigación, considerando que esta variable no se tuvo en cuenta en el estudio.

Igualmente, este estudio enfatiza en que la percepción de los problemas conductuales, desde el punto de vista de las madres y la educadora, corresponde a un juicio a partir de la percepción subjetiva de una o más personas acerca de la conducta del niño, relativizando el constructo y abriendo la posibilidad de conocer las distintas visiones acerca de la conducta de los niños. Así mismo, advierte sobre las diversas formas de funcionamiento de un individuo en diferentes contextos.

Por otro lado, para los psicólogos clínicos implica que la información que reciben está ligada a la observación de una persona y que es necesario complementar con observación del niño en otros contextos, como la casa y el jardín. Finalmente, para los investigadores es de gran relevancia tomar en cuenta el hecho de que el mismo instrumento arroja información muy distinta en las mismas dimensiones según el informante, lo cual es importante tener en consideración en futuras investigaciones.

\section{REFERENCIAS BIBLIOGRÁFICAS}

Achenbach, T. (2011). Commentary: Definitely More Than Measurement Error: But How Should We Understand and Deal With Informant Discrepancies? Journal of Clinical Child \& Adolescent Psychology, vol. 40, n. 1, 80-86.

Almonte, C., Montt, M. E. \& Correa, A. (2003). Psicopatología infantil y de la adolescencia. Santiago de Chile: Mediterráneo.

Briggs-Gowan, M. J., Carter, A., Bosson-Heenan, J., Guyer, A. \& Horwitz, S. (2006). Are Infanttoddler Social-emotional and Behavioral Problems Transient? Journal of the American Academy of Child and Adolescent Psychiatry, vol. 45, n. 7, 849-858.

Campbell, S. B., Shaw, D. S. \& Gilliom, M. (2000). Early Externalizing Behavior Problems: Toddlers and Pre-schoolers at Risk for Later Maladjustment. Development and Psychopathology, vol. 12, n. 3, 467-488.

Centro de Estudios del Desarrollo y Estimulación Psicosocial (CEDEP). (1983). Salud mental del escolar. Estandarización del inventario de problemas conductuales y destrezas sociales de T. Achenbach en niños de 6 a 11 años. Santiago de Chile: Publicación UNICEF.

De la Barra, F., Toledo, V. \& Rodríguez, J. (2002). Estudio de salud mental en dos cohortes de niños escolares de Santiago occidente. I: Prevalencia y seguimiento de problemas conductuales y cognitivos. Revista Chilena de Neuro-psiquiatría, vol. 40, n. 1, 9-21.

De los Reyes, A. (2011). Definitely More Than Measurement Error: But How Should We Understand and Deal With Informant Discrepancies? Journal of Clinical Child \& Adolescent Psychology, vol. 40, n. 1, 1-9.

., Youngstrom, E., Pabón, S., Youngstrom, J., Feeny, N. \& Findling, R. (2011). Internal Consistency and Associated Characteristics of Informant Discrepancies in Clinic Referred Youths Age 11 to 17 Years. Journal of Clinical Child \& Adolescent Psychology, vol. 40, n. 1, 36-53.

, A. \& Kazdin, A. E. (2004). Measuring Informant Discrepancies in Clinical Child Research. Psychological Assessment, vol. 16, n. 3, 330-334. 
Dirks, M., Boyle, M., \& Goergiades, K. (2011). Psychological Symptoms in Youth and Later Socioeconomic Functioning: Do Associations Vary by Informant? Journal of Clinical Child \& Adolescent Psychology, vol. 40, n. 1, 10-22.

Feeney-Kettler, K. A., Kratochwill, T. R. \& Kettler, R. J. (2011). Identification of Preschool Children at Risk for Emotional and Behavioral Disorders: Development and Validation of a Universal Screening System. Journal of School Psychology, vol. 49, n. 2, 197-216.

Hinshaw, S., Han, S., Erhardt, D. \& Huber, A. (1992). Internalizing and Externalizing Behavior Problems in Preschool Children: Correspondence among Parent and Teacher Ratings and Behavior Observations. Journal of Clinical Child Psychology, vol. 21, n. 2, 143-150.

Medina, C., Blas Navarro, J., Martinena, P., Baños, I., Vicens-Vilanova, J. \& Barrantes-Vidal, N. (2007). Evaluación de la conducta adolescente con las escalas de Achenbach: ¿Existe concordancia entre diferentes informantes? Salud Mental, vol. 30, n. 5, 33-39.

Mesman, J., Bongers, I. L. \& Koot, H. M. (2001). Preschool Developmental Pathways to Preadolescent Internalizing and Externalizing Problems. Journal of Child Psychological and Psychiatry, vol. 42, n. 5, 679-689.

Moffitt, T. \& Scott, S. (2008). Conduct Disorders of Childhood and Adolescence. In M. Rutter, D. Bishop, D. Pine, S. Scott, J. Stevenson, E. Taylor \& A. Thapar (Eds.), Rutter's Child and Adolescent Psychiatry (pp. 543-564). Massachusetts: Blackwell Publishing Limited.

Rioseco, P., Vicente, B., Valdivia, S., Cova, F., Melipillán, R. \& Rubi, P. (2009). Prevalencia de trastornos psiquiátricos en adolescentes infractores de ley. Estudio caso-control. Revista Chilena de Neuro-psiquiatría, vol. 47, n. 3, 190-200.

Rodríguez, S., Lira, M. I., Arancibia, V. \& Bralic, S. (2000). Inventario de problemas conductuales y socioemocionales para niños entre 3 y 5 años. Santiago de Chile: Universidad Católica de Chile.

Satake, H., Yoshida, K., Yamashita, H., Kinusawa, N. \& Takagishi, T. (2003). Agreement Between Parents and Teachers on Behavioral/Emotional Problems in Japanese School Children Using the Child Behavior Checklist. Child Psychiatry and Human Development, vol. 34, n. 2, 111-126.

Seguel, X., Edwards, M., De Amesti, A. \& Montenegro, H. (1992). Prevalencia de problemas conductuales y emocionales en la población preescolar de Santiago. Santiago: CEDEP.

Touliatos, J. \& Lindholm, B. (1981). Congruence of Parents and Teachers Ratings of Children Behavior Problems. Journal of Abnormal Child Psychology, vol. 9, n. 3, 436-354.

Winsler, A. \& Wallace, G. (2002). Behavior Problems and Social Skills Preschool Children: Parent-Teacher Agreement and Relations with Classroom Observation. Early Education \& Development, vol. 13, n. 1, 41-58. 
